\title{
ESPACIOS DEFINIDOS
}

\section{DEFINITE SPACES}

\author{
Héctor Carlos Guimaray Huerta ${ }^{1}$
}

RESUMEN

La matemática ha desarrollado, haciendo uso de axiomas, espacios matemáticos como espacios vectoriales, normados, métricos, topológicos, etc. El objetivo principal en este artículo es definir un espacio en el que las propiedades de un conjunto sean las mismas en los espacios mencionados anteriormente, para lo cual se considera una función conjunto a conjunto llamada función definida.

Palabras clave.- Espacio vectorial, Espacio topológico, Conjunto definido, Cápsula definida.

\section{ABSTRACT}

Mathematics has been developed, using axioms, mathematical spaces as vector spaces, normed, metric, topological and so on. The primary objective in this article is to define a space in which the properties of a set are the same in the spaces above, for which is considered a function set to set called defined function.

Key words.- Vector space, Topological space, Defined set, Defined hull.

\section{INTRODUCCIÓN}

En el espacio vectorial surgen los conceptos de subespacio vectorial, conjunto afín, conjunto convexo y cono, entre otros, los que a su vez dan lugar a cápsula lineal, afín, convexa y positiva respectivamente. Similarmente, en espacio topológico usando el concepto de conjunto cerrado consideramos la cápsula cerrada que no es otra cosa que la clausura de dicho conjunto. En el presente artículo se introduce el concepto de conjunto definido, cápsula definida para luego considerar el espacio definido con el objetivo de que las propiedades sean válidas en los espacios mencionados.

\section{PRELIMINARES}

\section{Subespacio vectorial}

Definición.- Sean $X$ un espacio vectorial sobre un campo $\mathrm{C}, \mathrm{A} \subseteq \mathrm{X}$.

Se dice que A es subespacio vectorial si: i) $x+y \in A \quad \forall x, y \in A$

ii) $\alpha \mathrm{x} \in \mathrm{A} \quad \forall \alpha \in \mathrm{C}, \mathrm{x} \in \mathrm{A}$ [5]

Proposición.- Sea $X$ un espacio vectorial sobre un campo C. Luego, $\bigcap A_{i}$ es un subespacio vectorial $\forall$ colección de subespacios vectoriales $\left\{\mathrm{A}_{\mathrm{i}}\right\}[1-5]$.

Definición:-- Sean X un espacio vectorial sobre un campo $\mathrm{C}, \mathrm{A} \subseteq \mathrm{X}$. Luego,

$<\mathrm{A}>=\left\{\mathrm{x} \in \mathrm{X} / \mathrm{x}=\sum_{\mathrm{k}=1}^{\mathrm{n}} \alpha_{\mathrm{k}} \mathrm{x}_{\mathrm{k}}, \mathrm{x}_{\mathrm{k}} \in \mathrm{A}, \alpha_{\mathrm{k}} \in \mathrm{C}\right\}$ se llama cápsula lineal de A. [4].

Proposición.- Sean $X$ un espacio vectorial sobre un campo $\mathrm{C} ; \mathrm{A}, \mathrm{B} \subseteq \mathrm{X}$. Luego:

i) $<\mathrm{A}>$ es un subespaciovectorial $\forall \mathrm{A} \subseteq \mathrm{X}[1]$

ii) $\mathrm{A} \subseteq \mathrm{B} \rightarrow<\mathrm{A}>\subseteq \leq<\mathrm{B}>$

iii) A es subespacio vectorial si y sólo si

$\mathrm{A}=\langle\mathrm{A}>$

\section{Conjunto afín}

Definición.- Sean X un espacio vectorial sobre un

${ }^{1}$ Maestro y Docente de la Facultad de Ciencias de la Universidad Nacional de Ingeniería, Lima, Perú 
campo $\mathrm{C}, \mathrm{A} \subseteq \mathrm{X}$. Se dice que $\mathrm{A}$ es conjunto afín si $\mathrm{A}=\mathrm{x}+\mathrm{S}$ donde $\mathrm{x} \in \mathrm{X}$ y $\mathrm{S}$ es un subespacio vectorial.

Proposición.- Sea $X$ un espacio vectorial sobre un campo C. Luego, $\cap \Lambda_{1}$ es un conjunto afín $\forall$ colección de conjuntos afines $\left\{\mathrm{A}_{\mathrm{i}}\right\}$.

Definición.- Sean $X$ un espacio vectorial sobre un campo $\mathrm{C}, \mathrm{A} \sqsubseteq \mathrm{X}$. Luego,

$\mathcal{A}(\mathrm{A})=\left\{\mathrm{x} \in \mathrm{X} / \mathrm{x}=\sum_{\mathrm{k}=1}^{\mathrm{n}} \alpha_{\mathrm{k}} \mathrm{x}_{\mathrm{k}}, \mathrm{x}_{\mathrm{k}} \in \mathrm{A}, \alpha_{\mathrm{k}} \in \mathrm{C}\right.$, $\left.\sum_{\mathrm{k}=1}^{\mathrm{n}} \alpha_{\mathrm{k}}=1\right\}$ se llama cápsula afín de A [7 y 8].

Proposición.- Sean $X$ un espacio vectorial sobre un campo $\mathrm{C} ; \mathrm{A}, \mathrm{B} \subseteq \mathrm{X}$. Luego:

$\forall \mathrm{A} \subseteq \mathrm{X}$

i) $\_l(\mathrm{~A})$ es un conjunto afín

ii) $\mathrm{A} \subseteq \mathrm{B} \Rightarrow \mathcal{A}(\mathrm{A}) \subseteq \mathcal{A}(\mathrm{B})$

iii) A es conjunto afín si y sólo si

$\mathrm{A}=\mathcal{A}(\mathrm{A})$

\section{Conjunto convexo}

Definición.- Sean X un espacio vectorial real, $\mathrm{A} \subseteq$ $X$. Se dice que $A$ es unconjunto convexo si $\lambda \mathrm{A}+(1-\lambda) \mathrm{A} \subseteq \mathrm{A} \quad \forall \lambda \in[0,1]$

Proposición.- Sea $X$ un espacio vectorial real. Luego, $\bigcap A_{-i}$ es un conjunto convexo $\forall$ colección de conjuntos convexos $\left\{\mathrm{A}_{\mathrm{i}}\right\}[6]$.

Definición.- Sean $X$ un espacio vectorial real, $\mathrm{A} \subseteq$ $X$. Luego, $[A]=\left\{x \in X / x=\sum_{k=1}^{n} \alpha_{k} x_{k}\right.$,

$\left.x_{k} \in A, \alpha_{k} \in C, \sum_{k=1}^{n} \alpha_{k}=1, \alpha_{k} \geq 0\right\}$ se llama cápsula convexa de $\mathrm{A}$.

Proposición.- Sean X un espacio vectorial real; A, $B \subset X$. Luego:

i) $[\mathrm{A}]$ es un conjunto convexo

$\forall \mathrm{A} \subseteq \mathrm{X}$

ii) $\mathrm{A} \subseteq \mathrm{B} \Rightarrow[\mathrm{A}] \bumpeq[\mathrm{B}]$

iii) A es conjunto convexo si y sólo si

$\mathrm{A}=[\mathrm{A}]$

\section{Cono convexo}

Definición.- Sean X un espacio vectorial real, A $\subset$ $X$. Se dice que A es uncono si $\lambda \mathrm{A} \subseteq \mathrm{A} \forall \lambda \geq 0$.

Se dice que $\mathrm{A} \subseteq \mathrm{X}$ es cono convexo si $\mathrm{A}$ es cono $\mathrm{y}$ convexo.

TECNIA 22 (2) 2012
Proposición.- Sea $X$ un espacio vectorial real. Luego, $\bigcap A_{i}$ es un cono convexo $\forall$ colección de conos convexos $\left\{\mathrm{A}_{\mathrm{i}}\right\}$.

Definición.- Sean $\mathrm{X}$ un espacio vectorial real, $\mathrm{A} \subseteq$ $\mathrm{X}$. Luego, $\mathcal{P}(\mathrm{A})=\left\{\mathrm{x} \in \mathrm{X} / \mathrm{x}=\sum_{\mathrm{k}=1}^{\mathrm{n}} a_{\mathrm{k}} \mathrm{x}_{\mathrm{k}}\right.$,

$\left.x_{\mathrm{k}} \in \mathrm{A}, \alpha_{\mathrm{k}} \in \mathrm{C}, \alpha_{\mathrm{k}} \geq 0\right\}$ se llama cápsula positiva de A.

Proposición.- Sean X un espacio vectorial real; A, $\mathrm{B} \subseteq \mathrm{X}$. Luego:

$\forall \mathrm{A} \subseteq \mathrm{X}$

i) $\mathcal{P}(\mathrm{A})$ es un cono convexo

ii) $\mathrm{A} \subseteq \mathrm{B} \Rightarrow \mathcal{P}(\mathrm{A}) \subseteq \mathcal{P}(\mathrm{B})$

iii) A es cono convexo si y sólo si

$\mathrm{A}=\mathcal{P}(\mathrm{A})[2 \mathrm{y} 8]$

\section{Conjunto cerrado}

Definición.- Sean $X$ un espacio topológico, $A \subseteq X$. Se dice que $\mathrm{A}$ es conjunto cerrado si $\mathrm{X} \backslash \mathrm{A}$ es abierto [7].

Proposición.- Sea X un espacio topológico. Luego, $\bigcap A_{i}$ es un conjunto cerrado $\forall$ colección de conjuntos cerrados $\left\{A_{i}\right\}$ en $X[7]$.

Definición.- Sean $\mathrm{X}$ un espacio topológico $\mathrm{A} \subseteq \mathrm{X}$. Luego, $\bar{A}=\{x \in X /$

$\mathrm{V} \cap A \mp \emptyset \mathrm{V}$ vecindad $\mathrm{V}$ de $\mathrm{x}\}$ se llama cápsula cerrada de A [3].

Proposición.- Sean X un espacio topológico; A, B ᄃ X. Luego:

$\forall \mathrm{A} \subseteq \mathrm{X}$

i) $\bar{A}$ es un conjunto cerrado

ii) $\mathrm{A} \subset \mathrm{B} \Rightarrow \overline{\mathrm{A}} \subset \overline{\mathrm{B}}$

iii) A es conjunto cerrado si y sólo si

$\mathrm{A}=\bar{A}[3]$.

\section{PROPUESTA DE INVESTIGACIÓN ESPACIO DEFINIDO}

Notación.- $\mathcal{P}(\mathrm{X})$ conjunto potencia de un conjunto $\mathrm{X}$.

Observación.- Conjunto definido es, por ejemplo, conjunto abierto, cerrado, subespacio vectorial, conjunto afín, convexo, etc. 
Definición.- Sea $X$ un conjunto dotado de un concepto de conjunto definido en $\mathrm{X}$ donde $\bigcap A_{i}$ es un conjunto definido $\forall$ colección de conjuntos definidos $\left\{A_{i}\right\}$ en $\mathrm{X}$,

$\mathrm{D}: \mathcal{T}(\mathrm{X}), \mathcal{T}(\mathrm{X})$ se llama función definida en $\mathrm{X}$ si:

\section{$\forall \mathrm{A} \subseteq \mathrm{X}$}

$$
\text { i) } \mathrm{D}(\mathrm{A}) \text { es conjunto definido }
$$

ii) $\mathrm{A} \subseteq \mathrm{B} \Rightarrow \mathrm{D}(\mathrm{A}) \subseteq \mathrm{D}(\mathrm{B})$

iii) A es conjunto definido si y sólo si $\mathrm{A}=\mathrm{D}(\mathrm{A})$

D (A) se llama cápsula definida de A

(X, D) se llama espacio definido

Ejemplos: $(\mathrm{X},<>),(\mathrm{X}, \mathcal{A}),(\mathrm{X},[\mathrm{]})$,

$(\mathrm{X}, \mathcal{P}),(\mathrm{X},-)$ expresadas anteriormente son espacios definidos.

Ejemplo: Sea X un espacio topológico dotado del concepto de conjunto abierto, int $\mathrm{A}$ el interior de A. Luego, (X, int) no es espacio definido.

\section{Solución}

$\underline{\text { Se tiene que.- }}$

i) int $\mathrm{A}$ es conjunto abierto $\forall \mathrm{A} \subseteq \mathrm{X}$

ii) $\mathrm{A} \subseteq \mathrm{B} \Rightarrow$ int $\mathrm{A} \subseteq$ int $\mathrm{B}$

iii) A es conjunto abierto si y sólo si

$\mathrm{A}=\operatorname{int} \mathrm{A}$

Sin embargo, $\| A_{i}$ no es un conjunto abierto $\forall$ colección de conjuntos abiertos.

$\left\{A_{i}\right\}$ en $X$

$\Longrightarrow$

(X, int) no es espacio definido.

\section{Resultados}

Proposición: $A \subseteq D(A)$

Prueba:

Sea $x \in A$

Ahora, sea $\left\{A_{i}\right\}$ una colección de conjuntos definidos en $\mathrm{X}$ donde $\mathrm{A} \subseteq \mathrm{A}_{\mathrm{i}}$.

\section{$\Rightarrow$}

$x \in A_{i} \forall i$

$\Rightarrow$

$\mathrm{x} \in \bigcap \mathrm{A}_{-\mathrm{i}}$ donde $\mathrm{A} \subseteq \mathrm{A}_{\mathrm{i}}$ con $\mathrm{A}_{\mathrm{i}}$ conjunto definido.

$$
\Rightarrow
$$$$
\mathrm{x} \in \mathrm{D}(\mathrm{A})
$$

$\Rightarrow$

$\mathrm{A} \subseteq \mathrm{D}(\mathrm{A})$

TECNIA 22 (2) 2012
Observación.- Considerando la proposición anterior se tiene:

$\mathrm{A} \subseteq<\mathrm{A}>$

$\mathrm{A} \subseteq \mathcal{A}(\mathrm{A})$

$\mathrm{A} \subseteq[\mathrm{A}]$

$\mathrm{A} \subseteq \mathcal{P}(\mathrm{A})$

$\mathrm{A} \subseteq \overline{\mathrm{A}}$

Proposición.- $\mathrm{D}(\mathrm{A})=\bigcap \mathrm{A}_{\mathrm{i}}$ donde $\mathrm{A} \subseteq \mathrm{A}_{\mathrm{i}}$ con $\mathrm{A}_{\mathrm{i}}$ conjunto definido.

Prueba:

$\mathrm{A} \subseteq \mathrm{A}_{\mathrm{i}}$

$\Rightarrow$

$\mathrm{A} \subseteq \cap \mathrm{A}_{\mathrm{i}}$

$\Rightarrow$

$\mathrm{D}(\mathrm{A}) \subseteq \mathrm{D}\left(\cap \mathrm{A}_{\mathrm{i}}\right)=\cap \mathrm{A}_{\mathrm{i}}$, por el axioma iii $)$

$\mathrm{D}(\mathrm{A}) \subseteq \bigcap \mathrm{A}_{\mathrm{i}}$

Por otra parte:

$\bigcap \mathrm{A}_{\mathrm{i}} \subseteq \mathrm{A}_{\mathrm{j}} \forall \mathrm{j}$

Se tiene que $\mathrm{A} \subseteq \mathrm{D}(\mathrm{A})$ con $\mathrm{D}(\mathrm{A})$ conjunto definido.

$\Rightarrow$ $\mathrm{D}(\mathrm{A})=\mathrm{A}_{\mathrm{j}}$, algún $\mathrm{j}$

$\Rightarrow$

$\bigcap \mathrm{A}_{\mathrm{i}} \subseteq \mathrm{D}(\mathrm{A})$

De (I) y (II):

$\mathrm{D}(\mathrm{A})=\bigcap \mathrm{A}_{\mathrm{i}}$ donde $\mathrm{A} \subseteq \mathrm{A}_{\mathrm{i}}$ con $\mathrm{A}_{\mathrm{i}}$ conjunto definido.

\section{CONCLUSIONES}

Considerando la proposición anterior se tiene:

$<\mathrm{A}>=\bigcap \mathrm{A}_{\mathrm{i}}$ donde $\mathrm{A} \subseteq \mathrm{A}_{\mathrm{i}}$ con $\mathrm{A}_{\mathrm{i}}$ subespacio vectorial.

$\mathcal{A}(\mathrm{A})=\cap \mathrm{A}_{\mathrm{i}}$ donde $\mathrm{A} \sqsubset \mathrm{A}_{\mathrm{i}}$ con $\mathrm{A}_{\mathrm{i}}$ conjunto afín.

$[\mathrm{A}]=\bigcap \mathrm{A}_{\mathrm{i}}$ donde $\mathrm{A} \subseteq \mathrm{A}_{\mathrm{i}}$ con $\mathrm{A}_{\mathrm{i}}$ conjunto convexo.

$\mathcal{P}(\mathrm{A})=\cap \mathrm{A}_{\mathrm{i}}$ donde $\mathrm{A} \subseteq \mathrm{A}_{\mathrm{i}}$ con $\mathrm{A}_{\mathrm{i}}$ cono convexo.

$\overline{\mathrm{A}}=\cap \mathrm{A}_{\mathrm{i}}$ donde $\mathrm{A} \subset \mathrm{A}_{\mathrm{i}}$ con $\mathrm{A}_{\mathrm{i}}$ conjunto cerrado. 
Es decir, usando el concepto introducido, hemos probado simultáneamente para subespacio vectorial, conjunto afín, conjunto convexo, cono convexo y conjunto cerrado; de igual manera para otros conceptos matemáticos siempre que cumplan los axiomas de la propuesta de investigación dada en el punto 3 .

\section{REFERENCIAS}

1. Howard, A., "Introducción al Álgebra Lineal", pp. 279, Editorial Limusa S. A., México, 2003.

2. Berge, C., "Topological Spaces", pp. 14, the Macmillan Company, New York, 1963.

3. Dugundji, J., "Topology", pp. 69, Allyn and Bacon, Inc., Boston, 1966.
4. Herstein, I. N., “Álgebra Moderna”, pp. 163, Editorial Trillas S. A. de C. V., México, 2002.

5. Hoffman, K., “Álgebra lineal”, pp. 34, 36, Editorial Dossat, S. A., Madrid, 1973.

6. Magaril-II'yaevG, G., "Convex Analysis: Theory and Applications", pp. 30, American Mathematical Society, USA, 2003.

7. Munkres, J. R., "Topología”, pp. 105, 106, Pearson Educación, S. A., Madrid, 2002.

8. Kusraev, A. G., Kitateladze, S. S., "Subdifferentials: Theory and Applications", pp. 3, 2 - 6, Kluwer Academic Publishers, Netherlands, 1995.

hguimaray@hotmail.com

Recepción de originales: enero 2013

Aceptación de originales: marzo 2013 